Article

\title{
Probing the Analytical Cancellation Factor of Short Scale Gravity Waves Using Na Lidar and Nightglow Data from the Andes Lidar Observatory
}

\author{
Fabio Vargas ${ }^{1, *,+}\left(\mathbb{D}\right.$, Javier Fuentes ${ }^{2,+}$, Pedro Vega ${ }^{3}$, Luis Navarro ${ }^{1}\left(\mathbb{D}\right.$ and Gary Swenson ${ }^{1}$ \\ 1 Remote Sensing \& Space Science Laboratory, Department of Electrical and Computer Engineering, \\ University of Illinois at Urbana-Champaign, Urbana, IL 61801, USA; lnav@illinois.edu (L.N.); \\ swenson1@illinois.edu (G.S.) \\ 2 Gemini Observatory Southern Operations Center, Av. Juan Cisternas 1500, c/o AURA casilla 603, \\ La Serena 1700000, Chile; jfuentes@gemini.edu \\ 3 Physics Department, Faculty of Science, University of La Serena, Av. Juan Cisternas 1200, \\ La Serena 1700000, Chile; pvega@userena.cl \\ * Correspondence: fvargas@illinois.edu \\ + These authors contributed equally to this work.
}

Received: 21 October 2020; Accepted: 30 November 2020; Published: 3 December 2020

\begin{abstract}
The cancellation factor (CF) is a model for the ratio between gravity wave perturbations in the nightglow intensity to those in the ambient temperature. The CF model allows us to estimate the momentum and energy flux of gravity waves seen in nightglow images, as well as the divergence of these fluxes due to waves propagating through the mesosphere and lower thermosphere region, where the nightglow and the Na layers are located. This study uses a set of wind/temperature $\mathrm{Na}$ lidar data and zenith nightglow image observations of the $O H$ and $O\left({ }^{1} S\right)$ emissions to test and validate the CF model from the experimental perspective. The dataset analyzed was obtained during campaigns carried out at the Andes Lidar Observatory (ALO), Chile, in 2015, 2016, and 2017. The modeled CF was compared with observed CF values calculated using the ratio of wave amplitude in nightglow images to that seen in lidar temperatures for vertically propagating waves. We show that, in general, the modeled CF underestimates the observed CF results. However, the $O\left({ }^{1} S\right)$ emission line has better agreement with respect to the modeled value due to its supposedly simpler nightglow photochemistry. In contrast, the observed $\mathrm{CF}$ for the $\mathrm{OH}$ emission deviates by a factor of two from the modeled CF asymptotic value.
\end{abstract}

Keywords: airglow; all-sky imagery; atmospheric gravity waves; cancellation factor; lidar; mesosphere-lower thermosphere

\section{Introduction}

Propagating atmospheric gravity waves (AGWs) perturb the density and composition of major [1,2] and minor [3-5] species in the mesosphere and lower thermosphere (MLT). These species are part of a chain of complex chemical reactions occurring as a result of solar ultraviolet radiation, which ultimately leads to nightglow emissions [6-9].

The mesospheric nightglow layers are excellent tracers of mesopause dynamics and have been used to study the wave-induced perturbations in atmospheric density, temperature, and winds [10,11]. In the presence of a propagating AGW, the mesospheric nightglows are influenced by perturbations in all the species playing important roles in their chemistry, by perturbations in temperature that affect chemical reaction rates, as well as by the redistribution of the background $\mathrm{O}$ and $\mathrm{O}_{3}$ profiles caused by wave dynamics alone that are important for the final nightglow layer shapes and brightness. 
For instance, studies of $\mathrm{OH}^{*}$ explain the differences in the magnitude of the wave perturbations as well as in the observed wave phases in $\mathrm{OH}^{*}$ brightness and rotational temperature through the Krassovsky ratio [12], which may depend on the gravity wave amplitudes, periods, and wavelengths [13-17].

Airglow emission brightness fluctuations have been the focus of different studies using one-dimensional models upon certain atmosphere conditions for gravity waves with various intrinsic parameters and damping rates (e.g., [18-21]). These studies define the ratio of the relative perturbation in intensity $\frac{I^{\prime}}{\bar{I}}$ to that in ambient temperature $\frac{T^{\prime}}{\bar{T}}$ as the cancellation factor $\left(C F=\frac{I^{\prime} / \bar{I}}{T^{\prime} / \bar{T}}\right)$, which depends on the wave amplitude within a wave cycle and the vertical wavelength. CF is used to determine how the layer responds to wave perturbations of various vertical scales, and is most useful in the determination of the flux of momentum and energy and flux divergences of waves seen in the nightglow $[19,21]$. The motivation to study wave-induced momentum flux and wave-induced momentum flux divergence in the mesosphere also relies on the fact that waves present in nightglow images are usually dominated by quasi-monochromatic oscillations with large vertical wavelengths [19], periods $<1 \mathrm{~h}$, and phase speeds from $40-70 \mathrm{~m} / \mathrm{s}$ [22,23], and are responsible for $75 \%$ of wave energy content in the mesosphere [24], causing decelerations of $\sim 100 \mathrm{~ms}^{-1} /$ day near the mesopause [25].

An analytical expression for $\mathrm{CF}$ was first derived by [18] for the $\mathrm{OH}(8,3)$ nightglow brightness, which is defined as the height integral over the layer's volume emission rate (VER), relating this brightness to the temperature perturbation at the altitude of maximum VER. The CF expression from [18] was used in [19] to relate measurements of gravity wave energy and momentum flux in several instruments. In [20] the modeling study was extended for the $\mathrm{O}_{2}(b)$ atmospheric bands, allowing investigation of the relations between the amplitude and phase of the nightglow perturbations induced by gravity waves from simultaneous measurements in the $\mathrm{OH}(8,3)$ and $\mathrm{O}_{2}(b)$ layers. Finally, [21] presented a comprehensible one-dimensional model adding the $O\left({ }^{1} S\right)$ emission line to the study of nightglow emission in response to AGW perturbations. The goal was to explore the vertical flux of horizontal momentum and wave effects on the atmosphere from the three $O\left({ }^{1} S\right)$, $\mathrm{OH}(8,3)$, and $\mathrm{O}_{2}(b)$ nightglow layers. The latter study drove the motivation to derive the uncertainties in momentum flux and accelerations due to gravity wave parameters estimated from mesospheric nightglow emissions reported in [26].

In this study, we present the first empirical assessment of the cancellation factor using $\mathrm{Na}$ lidar data and nightglow all-sky imagery of the $\mathrm{OH}(6,2)$ and $O\left({ }^{1} \mathrm{~S}\right)$ emissions during observing campaigns during 2015, 2016, and 2017 at the Andes Lidar Observatory (ALO), Cerro Pachón, Chile. We provide the magnitude of $\mathrm{CF}$ for multiple waves detected during these campaigns and directly compare these observations with the modeled CF as presented by [21].

\section{Instrumentation and Methodology}

ALO is a facility for middle- and upper-atmosphere studies and is located at $\left(30.3^{\circ} \mathrm{S}, 70.7^{\circ} \mathrm{W}\right)$ at an altitude of $2530 \mathrm{~m}$ near to Cerro Pachón, Chile. The ALO facility was designed to investigate wave dynamics, including the influence of mountain waves in the MLT region. It is equipped with a suite of optical instruments including an Na resonance lidar (acronym for LIght Detection And Range), and all-sky nightglow imagers. ALO also houses a static meteor radar, a mesospheric temperature mapper (MTM) camera, an aerospace narrow field of view cryogenic camera, and a GPS receiver.

The ALO all-sky imager records nightglows of hydroxyl $\mathrm{OH}$ Meinel bands and atomic oxygen line emissions (Figure 1). The imager ASI-1 collects the nightglow emissions using the instrumental configuration presented in Table 1. The ASI-1 images present a signal-to-noise ratio of better than 10 for the $\mathrm{OH}$ filter for image acquisition carried out during summer when the nightglow brightness is fainter. 


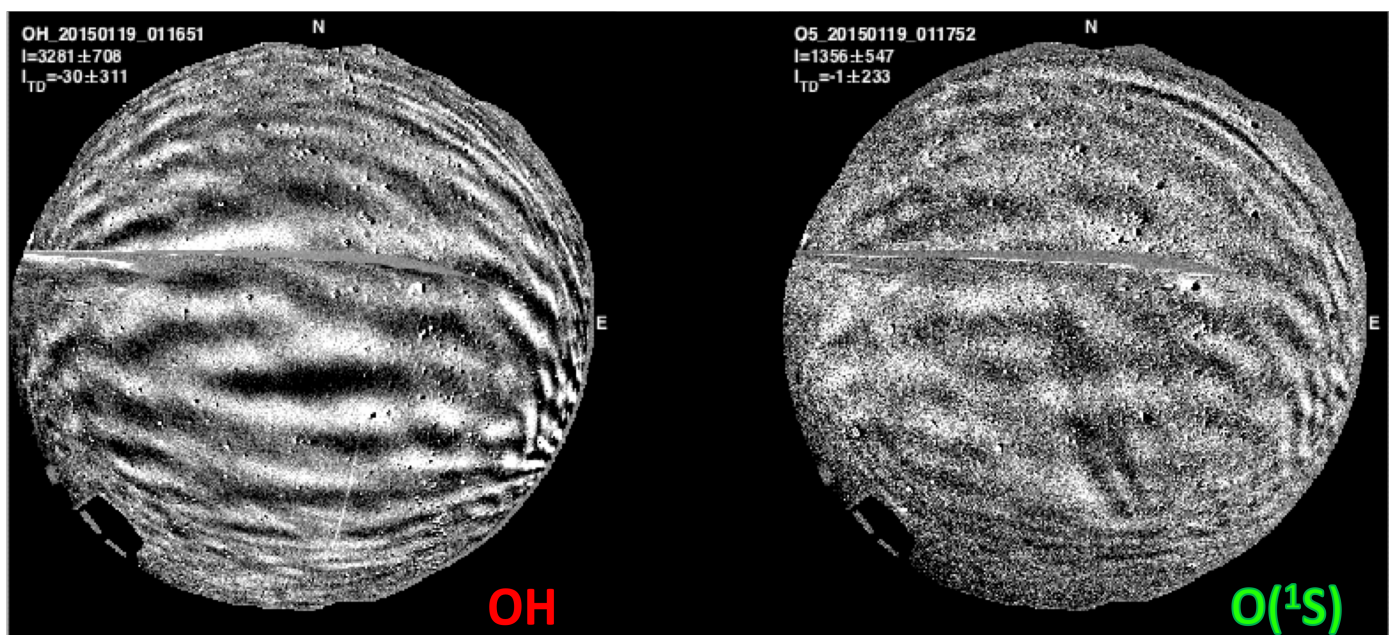

Figure 1. $O H($ left $)$ and $O\left({ }^{1} S\right)$ (right) nightglow emission images taken on 19 January 2015 at 0116 UTC and 0117 UTC. These images were not preprocessed to show the fisheye lens distortion of the night sky. Without preprocessing, the image field of view is about $1500 \mathrm{~km}^{2}$. Both images were captured with the Andes Lidar Observatory (ALO) all-sky imaging system (ASI-1).

Table 1. ALO nightglow imager (ASI-1) filter wheel configuration along with the full width at half maximum (FWHM) and exposure time. The $O\left({ }^{1} S\right)$ and $O H(6,2)$ filters were used here to estimate wave amplitudes in airglow intensity.

\begin{tabular}{lccc}
\hline Filter & $\lambda_{\text {center }}(\mathbf{n m})$ & FWHM (nm) & Exp.Time (s) \\
\hline$\left.B G_{O(1} S\right)$ & 551.0 & 3 & 90 \\
$O\left({ }^{1} S\right)$ & 557.7 & 3 & 90 \\
$O\left({ }^{1} \mathrm{D}\right)$ & 630.0 & 3 & 75 \\
$O H(6,2)$ & 840.0 & 20 & 60 \\
$\mathrm{O}_{2}(0,1)$ & 866.0 & 7 & 45 \\
\hline
\end{tabular}

The ALO lidar system transmits a nominal power of $1.5 \mathrm{~W}$ to obtain temperature, wind velocity, and Na density profiles typically at resolution of $1 \mathrm{~min}, 500 \mathrm{~m}$ between 80 and $105 \mathrm{~km}$. The laser is a source of coherent light locked at the $\mathrm{Na}$ resonance frequency at the D2a line. This central frequency $\left(f_{o}\right)$ is shifted by $\pm 630 \mathrm{MHz}$ to obtain the shifted frequencies $\mathrm{f}_{+}$and $\mathrm{f}_{-}$in a sequence to produce the optical excitation of the mesospheric sodium layer around the Na D2a line-width, enabling the production of an artificial beacon source. The temperature and line-of-sight wind are derived based on the ratios among the back-scattered signals at these three frequencies, as described in [27]. The $\mathrm{Na}$ lidar is operated in zenith and off-zenith modes to measure the wind and temperature using the three-frequency technique (see [28]). The integration time for lidar scans varies between campaigns from 60 to $90 \mathrm{sec}$ for each direction (zenith, south, east), which depends on the signal-to-noise ratio retrieved from the photon returns. As an example of the ALO lidar system capability, wind and temperature measurements versus time and altitude are shown in Figure 2, although Na density and vertical wind velocity can still be estimated directly from ALO lidar scans.

Observations using the Na lidar and nightglow imagery system are carried out in low Moon periods throughout the year. All-sky images of the nightglow are taken simultaneously with the lidar, and individual gravity wave occurrence in both systems can be monitored during the observation time. The observations used in this study are summarized in Tables 2 and 3. The imagery and lidar datasets were obtained at ALO during campaigns carried out during 2015, 2016, and 2017. 

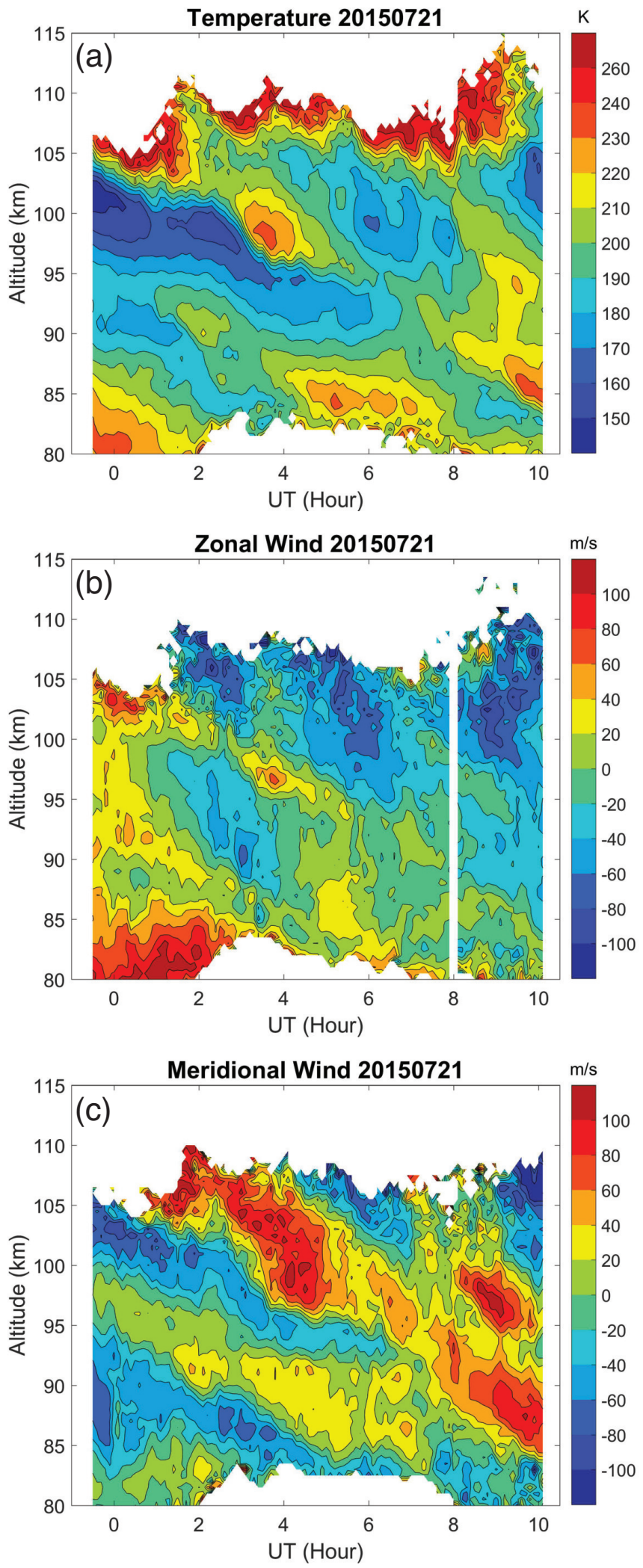

Figure 2. Lidar measurements taken on 21 July 2015 of (a) temperature, (b) zonal wind, and (c) meridional wind over ALO in Cerro Pachón, Chile. Note the nonlinear contour scale (at lowest altitudes) is used to highlight the low sensitivity of the $\mathrm{Na}$ winds in the mesosphere. 
Table 2. Imaging dataset and the number of prominent atmospheric gravity waves (AGWs) detected from the observing campaigns corresponding to 2015, 2016, and 2017 for the $O H$ and $O\left({ }^{1} S\right)$ emission lines.

\begin{tabular}{lllccc}
\hline Year & Month & Day & \# Nights & \# AGW $(\boldsymbol{O H})$ & \# AGW $\boldsymbol{O}\left({ }^{\mathbf{S}} \boldsymbol{)}\right)$ \\
\hline 2015 & Jan-Feb & $27-30,02$ & 5 & 2355 & 915 \\
2015 & April & $17-25$ & 8 & 3515 & 86 \\
2015 & July & $14-25$ & 11 & 2890 & 2116 \\
2015 & November & $01-08$ & 7 & 200 & 31 \\
\hline & 4 campaigns & & 31 & 8960 & 3145 \\
\hline 2016 & Feb-Mar & $25-29,01-15$ & 19 & 4435 & 885 \\
2016 & June & $06-11$ & 6 & 2710 & 1505 \\
2016 & Oct-Nov & $23-31,01-09$ & 17 & 225 & 60 \\
\hline & 3 campaigns & & 42 & 7370 & 2450 \\
\hline 2017 & April & $21-29$ & 8 & 2060 & 2405 \\
2017 & November & $20-28$ & 9 & 95 & 60 \\
2017 & December & $12-22$ & 10 & 515 & 70 \\
\hline & 3 campaigns & & 27 & 2670 & 2535 \\
\hline Total & 10 campaigns & & 100 & 19,000 & 8130 \\
\hline
\end{tabular}

Table 3. Lidar dataset for each operation period being covered by the image acquisition at ALO.

\begin{tabular}{llcccc}
\hline Year & Month, Day & \# Nights & \# Hours & ${\text { Nights with Winds }\left(\mathrm{U}^{\boldsymbol{a}}, \mathrm{V}^{\boldsymbol{b}}\right)}_{\text {Average CPS }^{\boldsymbol{c}}}$ \\
\hline 2015 & Jan-Feb (16-31,01-02) & 16 & 96.4 & 5 & 559 \\
2015 & April (15-29) & 14 & 101.9 & 8 & 556 \\
2015 & July (14-25) & 11 & 65.3 & 11 & 554 \\
2015 & November (27-30,01-08) & 8 & 69.6 & 7 & 700 \\
\hline 2016 & Feb-Mar (25-29,01-15) & 19 & 96.7 & 19 & 540 \\
2016 & June (06-11) & 6 & 66.0 & 6 & 760 \\
2016 & Oct-Nov (23-31,01-04) & 17 & 91.4 & 17 & 582 \\
\hline 2017 & April (21-29) & 8 & 50.8 & 8 & 609 \\
2017 & November (20-28) & 9 & 57.0 & 9 & 299 \\
2017 & December (12-24) & 12 & 70.7 & 10 & 213 \\
\hline Total & 10 campaigns & 155 & 1043.9 & 100 & 7174 \\
\hline
\end{tabular}

${ }^{a}$ : U represents the zonal winds. ${ }^{b}: \mathrm{V}$ represents the meridional winds. ${ }^{c}:$ counts per shot (CPS); the units of this measurement are photons $/ \mathrm{cm}^{2} / \mathrm{s} / \mathrm{W}$ of the propagating laser power; raw photon count data are processed off-line and preliminary results are shown at the following link: http://lidar.erau.edu/data/nalidar/index. php.

The modeled CF used here for comparison with our observational data was derived in [21] using a linear, one-dimensional model to describe the temporal and spatial variability of the volume emission rate (VER) of a nightglow emission in response to AGW perturbations. The photochemistry involved in the processes leading to $O\left({ }^{1} S\right)$ production and the $\mathrm{OH}$ Meinel band spectrum, as well as the intensity and weighted temperature due to upward propagating AGWs, is also described in [21].

In order to maintain the solutions in the linear range, a number of assumptions were considered in the model including, for instance, that wave amplitudes are small $(<1 \%)$ so that the AGW linear theory can be used to describe waves via the polarization and dispersion relationships. The wave perturbation of $1 \%$ amplitude is defined only in temperature at a reference altitude of $z_{r}=75 \mathrm{~km}$. Once the model considers only saturated waves, the wave amplitude does not change within the altitude range. The background atmosphere specified by the MSIS00 model is unchanged by the waves (e.g., [29]). A windless atmosphere (no shear with altitude) where the waves are propagating vertically through the layers was also considered in the [21] model. The simulations consisted of launching 
a gravity wave with $T^{\prime} / \bar{T}=1 \%$ at $\mathrm{z}_{r}$, and then varying its vertical wavelength, $\lambda_{z}$, and damping coefficient, $\beta$, in each iteration of the model. The resulting wave-perturbed nightglow VER and the intrinsic parameters of the simulated wave are then recorded for further analysis and fitting.

In addition, observed CF values were calculated for each individual gravity wave detected by our nightglow imager and lidar systems. Our methodology analyzes the perturbations of waves in nightglow images and also in lidar temperature in the vicinity of the nominal nightglow peak altitude. The observed $\mathrm{CF}$, calculated using the observed perturbations, is defined for the nightglow intensity as $C F=A_{I} / A_{T}$. Here, $A_{I}=I^{\prime} / \bar{I}$ and $A_{T}=T^{\prime} / \bar{T}$, where primed quantities refer to the wave fluctuation and bar quantities to the unperturbed background. $A_{I}$ is obtained from $O H$ and $O\left({ }^{1} S\right)$ nightglow image processing, and $A_{T}$ from the lidar temperature data at the time of wave perturbation occurrence in the nightglow. The background temperature is estimated from the lidar measurements around the wave event occurrence time as the average temperature around \pm 2 wave periods. The observed $C F$ is then compared to the CF model of [21].

We have estimated the intrinsic wave parameters from the image dataset, such as the horizontal wavelength $\left(\lambda_{h}\right)$, wave orientation $(\theta)$, wave phase $(\phi)$, wave period $(\tau)$, horizontal phase velocity $(c)$, and the relative wave amplitude $\left(A_{I}=I^{\prime} / \bar{I}\right)$, by performing the usual preprocessing routines (i.e., dewarping, star removal, coordinate transformation, detrending, and filtering as described in [30]). At the preprocessing stage, each individual image is mapped onto a uniform $512 \times 512 \mathrm{~km}^{2}$ grid of pixels in geographical coordinates with a resolution of $1 \mathrm{~km} /$ pixel. The assumed altitudes for the $\mathrm{OH}$ and $O\left({ }^{1} S\right)$ emissions were 88 and $95 \mathrm{~km}$, respectively. The integration times used in this study were $60 \mathrm{~s}$ for the $\mathrm{OH}$ and $90 \mathrm{~s}$ for the $O\left({ }^{1} \mathrm{~S}\right)$. In particular, we used mean horizontal winds from the lidar to perform Doppler correction of wave periods.

The wave amplitude is obtained from the magnitude of the dominant peaks of the cross-spectrum of time-difference nightglow images. Only dominant wavenumber peaks with energy larger than $10 \%$ of the total spectrogram energy are considered as legitimate waves. This is determined from a series of three consecutive nightglow images materializing in the 2D-FFT amplitude cross-periodogram as prominent peaks. We only use a small area of the image to determine the wave amplitude as the fields of view of the imager and lidar are different. This small portion is a $172 \times 172 \mathrm{~km}^{2}$ window centered at zenith. The lidar beam falls at the center of this window, at the image zenith. The wave amplitude in lidar temperature is estimated in time around the instant of occurrence of the wave observed in the nightglow images within a time window of \pm 2 wave periods around the occurrence time. For instance, if the wave period is $10 \mathrm{~min}$, the time window is $40 \mathrm{~min}$ long. Hence, the wave amplitude $I^{\prime} / \bar{I}$ is determined spatially, while the wave amplitude $T^{\prime} / \bar{T}$ is determined temporally. This procedure is valid as the wave amplitude is independent of either the temporal or spatial coordinates.

In order to compute the temperature perturbations $A_{T}=T^{\prime} / \bar{T}$ from lidar, we have removed the mean $(\bar{T})$ of each temperature altitude to determine $T^{\prime}=T-\bar{T}$ corresponding to the nominal altitude of the observed nightglows. Because we estimate the wave amplitude in temperature around the wave occurrence time, we assume the temperature perturbation is due to the same wave seen in the nightglow. Then, after selecting short wave periods $(\tau<1 \mathrm{~h})$ from prominent gravity wave events detected in imaging data, we estimate the observed CF for the two nightglow emissions for these prominent waves.

The wave amplitude in $\mathrm{T}$ for each nightglow layer is obtained at $z_{r}$ representing the altitude of the layer. Again, $I^{\prime}$ and $\bar{I}$ are the perturbed and unperturbed nightglow intensity from the images, respectively, while $T^{\prime}$ and $\bar{T}$ represent the perturbed temperature the unperturbed temperature obtained at the nominal altitudes of the observed nightglows, respectively. The ratio between $A_{I}=I^{\prime} / \bar{I}$ and $A_{T}=T^{\prime} / \bar{T}$ perturbations is our experimental estimation of the magnitude of the CF. The range of the relative amplitudes in temperature $A_{T}$ and nightglow intensity $A_{I}$ has been chosen so it does not break the linearity of the solutions in the modeled CF. This way, the dispersion and polarization equations remain valid throughout the analysis. 
We have also defined cutoff limits for filtering out waves presenting parameters not consistent with the modeled CF (Table 4). This way, wave amplitudes obtained from the image processing are comparable to the model. Finally, we have taken the relative wave and temperature intensities to compare the observed cancellation factor against the modeled CF relationship in [21]. The uncertainties shown in this paper have been derived by using Equation (11), the fitting coefficients presented in Table 1, and Equation (12) of [26].

Table 4. Criteria used for filtering the dataset for the $O H$ Meinel band and $O\left({ }^{1} S\right)$ emission line.

\begin{tabular}{ccccccc}
\hline Emission & $\mathrm{z}_{\boldsymbol{r}} \mathbf{( \mathbf { k m } )}$ & $\boldsymbol{I}^{\prime} / \overline{\boldsymbol{I}}$ & $\boldsymbol{T}^{\prime} / \overline{\mathbf{T}}$ & CF Magnitude & $\lambda_{\boldsymbol{z}}(\mathbf{k m})$ & $\boldsymbol{\tau}$ (min) \\
\hline$O H$ & 88 & $\geq 3$ & $0.75-1.25$ & $\leq 10$ & $14-60$ & $\geq 12$ \\
$O\left({ }^{1} S\right)$ & 95 & $\geq 4$ & $0.75-1.25$ & $\leq 10$ & $10-60$ & $\geq 12$ \\
\hline
\end{tabular}

\section{Results}

Prominent AGWs from the image processing were observed in 85 and 60 out of 100 nights of the initial sample for the $O H(6,2)$ Meinel band emission and $O\left({ }^{1} S\right)$ emission line, respectively. After filtering the prominent wave events presented in Table 2 using the criteria in Table 4, 94 wave events remained on 11 nights in 2015, 113 waves through 19 nights in 2016, and 30 waves on 4 nights in 2017 campaigns associated with the $O H$ band emission. Following the same filtering procedure for the $O\left({ }^{1} S\right)$ emission line, 43 wave events remained for 9 nights in 2015, 50 waves for 9 nights in 2016, and 98 waves for 5 nights in 2017. Table 5 summarizes these results for comparison purposes.

Figure 3 also shows the modeled CF error (dashed thin lines) that is dominated by the assigned error in $\lambda_{z}$ of $\sim 17 \%$. This assigned error is the average of the error in the vertical wavelength of AGWs from nightglow observations. However, small errors in the modeled CF fitting coefficients also contribute to the overall error in the modeled CF. In addition, the observed CF error is calculated for each wave event using the methodology of [26]. There are uncertainties in each wave parameter estimated from the nightglow observations that are transferred to the observed CF of each individual wave. These uncertainties also depend on the altitude of each emission. Observe that the errors in the individual observed CF values $\left(\sigma_{\lambda_{z}}\right)$ and their associated vertical wavelength is shown in Figure 3, which are estimated from Equations (8) and (12) of [26]. It is expected that the relative uncertainty in $\mathrm{CF}\left(\sigma_{C F}\right)$ is a function of $\lambda_{z}$ and increases as $\lambda_{z}$ decreases.

Table 5. Number of wave events for the $O H$ and $O\left({ }^{1} S\right)$ emissions after applying the criteria in Table 4. These quantities represent the filtered image dataset for the cancellation factor $(\mathrm{CF})$ computation taken during the observing runs presented in Tables 2 and 3.

\begin{tabular}{ccccc}
\hline Years & $\boldsymbol{O H}$ & $\boldsymbol{O}\left({ }^{\mathbf{S}} \boldsymbol{S}\right)$ & \# Nights $\boldsymbol{O H}$ & \# Nights $\boldsymbol{O}\left({ }^{\mathbf{S}} \boldsymbol{S}\right)$ \\
\hline 2015 & 94 & 43 & 11 & 9 \\
2016 & 113 & 50 & 19 & 9 \\
2017 & 30 & 98 & 4 & 5 \\
\hline Total & 237 & 191 & 34 & 23 \\
\hline
\end{tabular}

The observed CF values are estimated for both $O H(6,2)$ and $O\left({ }^{1} S\right)$ emission during 2015, 2016, and 2017 as shown in Figure 3. The measurement of CF values for the $O H$ emission deviates from the theoretical CF relationship (black continuous lines) more than $O\left({ }^{1} S\right)$, showing that the $O\left({ }^{1} S\right)$ has better agreement with the modeled CF in the range $\lambda_{z} \sim 20-60 \mathrm{~km}$. 

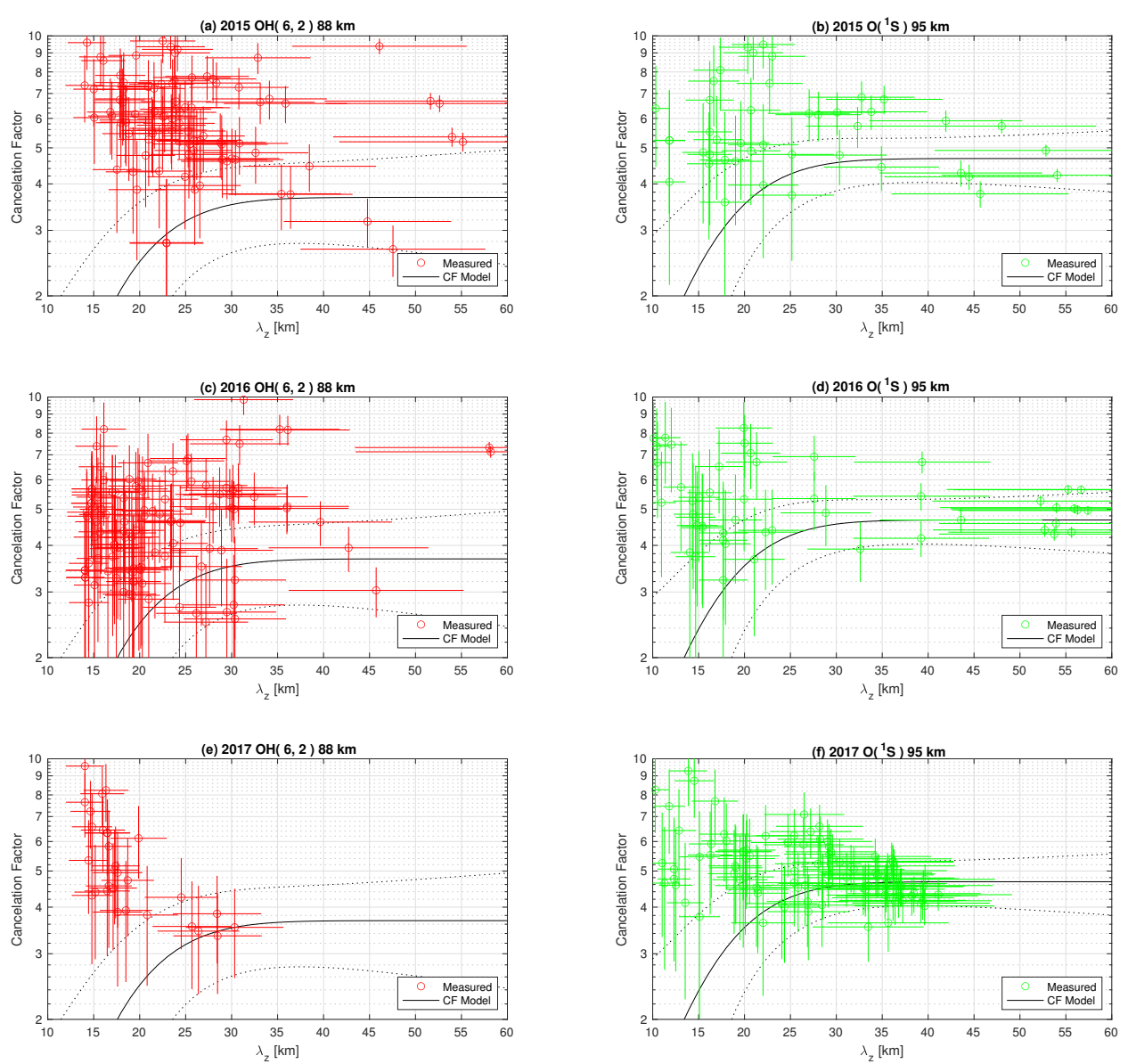

Figure 3. Observed CF for $O H$ (red open circles) during (a) 2015, (c) 2016, and (e) 2017, and for $O\left({ }^{1} S\right.$ ) (green open circles) during during (b) 2015, (d) 2016, and (f) 2017. The horizontal $\left(\sigma_{\lambda_{z}}\right)$ and vertical $\left(\sigma_{C F}\right)$ errors are also shown. The dotted thin lines denote the $95 \%$ confidence bounds $(2 \sigma)$ around the analytic curves shown as the continuous black lines in the plots.

The uncertainties have been derived for $\lambda_{z}$ at the $O H$ and $O\left({ }^{1} S\right)$ emission altitudes. The average values are $\sigma_{\lambda_{z}} \sim 16 \%$ and $\sigma_{\lambda_{z}} \sim 17 \%$ for the $O H$ and $O\left({ }^{1} S\right)$ emissions, respectively. Reference [26] found that $\lambda_{z}$ shows uncertainties of $\sim 10 \%$ and $8 \%$ for $O H$ and $O\left({ }^{1} S\right)$ emissions. The estimated uncertainties in observed CF are $\sigma_{C F} \sim 10 \%$ for $\mathrm{OH}$ emission and $\sigma_{C F} \sim 7 \%$ for the green line $O\left({ }^{1} \mathrm{~S}\right)$, respectively. The dotted thin lines in Figure 3 represent the 95\% confidence levels derived for the modeled CF. The uncertainties for both emissions range between 15 and $24 \%$, and are higher for shorter $\lambda_{z}$ as expected.

Some observed CF data points fall within the modeled CF confidence levels (dashed thin lines) for the $\mathrm{OH}$ emission (comparable to the full sample), which indicates those points are in agreement with the modeled CF relationship. To estimate how far the data points fall from the modeled CF curve, we have built histograms and kernel density estimators (KDEs) for the samples. Figure 4 shows the residuals between the observed and modeled CF data points. The samples have been filtered out using a 3 times the standard deviation criteria to remove outliers.

The KDE curves (solid red line in Figure 4) show the density plot as a smoother version of the histograms. The histograms are normalized by default so that they have the same y-scale as the density plots. In addition, we fitted a Gaussian function with bin width following the Freedman-Diaconis rule [31], which changes the distribution drawn at each data point and the overall distribution. However, we have decided to use the Gaussian kernel density estimation to compute the mean values for both normal distributions. 
The histograms in Figure 4 have a well-defined central tendency in the normal distribution for both $\mathrm{OH}$ and $O\left({ }^{1} \mathrm{~S}\right)$ emissions. The center of the $\mathrm{CF}_{\mathrm{O}\left({ }^{1} S\right)}$ is closer to zero than $\mathrm{CF}_{\mathrm{OH}}$ according to the mean value of the Gaussian curves. The peak of the distribution for both emissions is found to be skewed to the right, meaning that the model underestimates the observed values. The arithmetic mean values have been derived for the $O H$ and $O\left({ }^{1} S\right)$ emissions as $\mu_{O H}=3.1$ and $\mu_{O\left({ }^{1} S\right)}=1.42$, respectively.
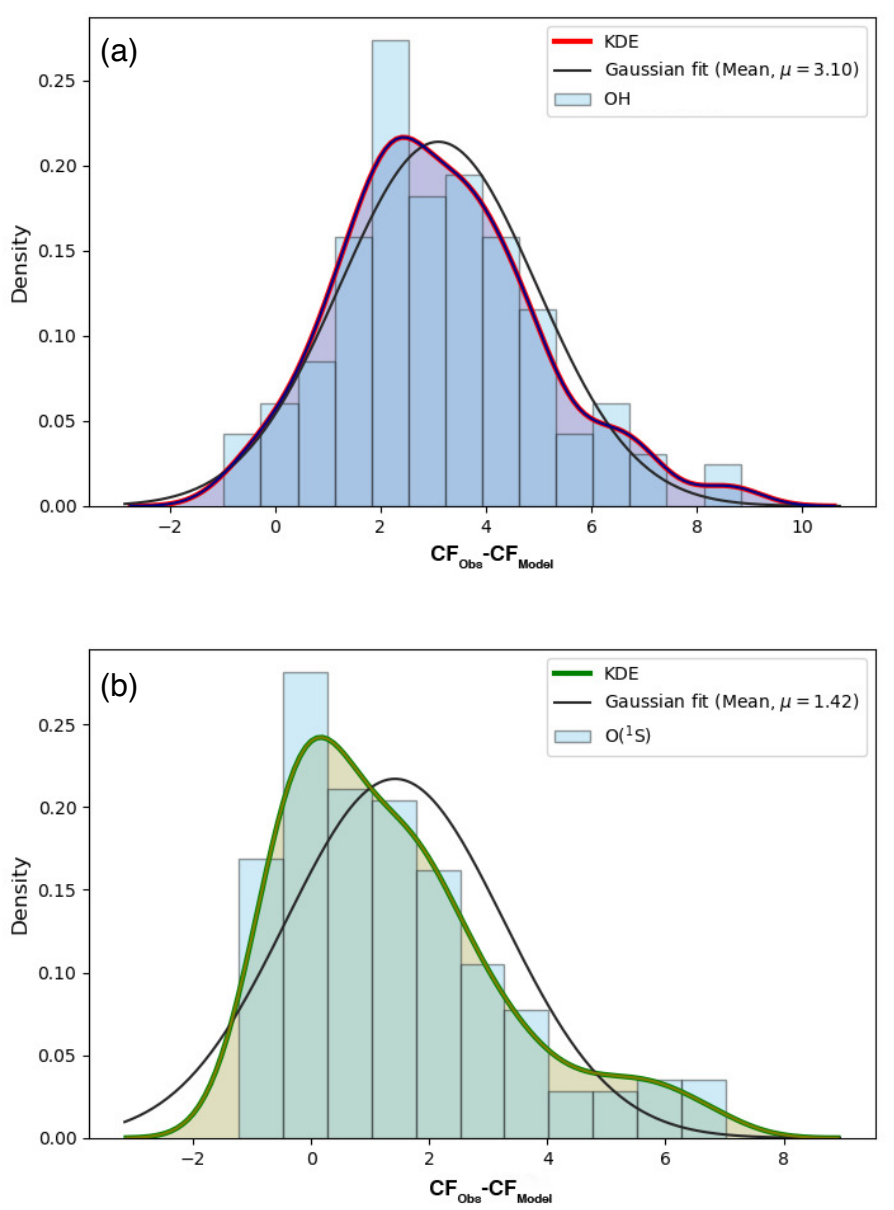

Figure 4. Histograms of the residuals and density plots for the (a) $O H$ and (b) $O\left({ }^{1} S\right)$ emissions.

The main contribution of this work is to test the modeled CF relationship using the observed data. From that, we have verified that the theoretical model underestimates the observations. It is important to measure this discrepancy to make corrections to the theoretical relationship for both emissions. To do so, we have evaluated the discrepancy between observed and modeled CFs, and added them to the corresponding modeled CF for each layer to obtain corrected predictions. To estimate the discrepancy, we use the weighted mean and the standard deviation of the mean:

$$
\overline{C F}=\frac{\sum_{i=1}^{n} \frac{C F_{i}}{\sigma_{C F_{i}}^{2}}}{\sum_{i=1}^{n} \frac{1}{\sigma_{C F_{i}}^{2}}}
$$

and

$$
\sigma_{\overline{\mathrm{CF}}}=\frac{1}{\sqrt{\sum_{i=1}^{n} \frac{1}{\sigma_{C F_{i}}^{2}}}}
$$


We use the weighted mean and standard deviation as they take into account the spread in the data (Table 6). Data points presenting smaller uncertainties $\left(\sigma_{C F}\right)$, that is, higher accuracy measurements, will have a larger influence on the weighted mean. This is better than using the arithmetical mean and standard deviation that just ignore the magnitude of the error of each measurement.

Table 6. Magnitude of the weighted mean and standard deviation for $O H$ and $O\left({ }^{1} S\right)$ emissions.

\begin{tabular}{ccccc}
\hline Year & $\overline{C F}_{\mathrm{OH}}$ & $\sigma_{\overline{\mathrm{CF}}_{\mathrm{OH}}}$ & $\overline{\mathrm{CF}}_{\boldsymbol{O}\left({ }^{1} S\right)}$ & $\sigma_{\overline{\overline{C F}}_{\boldsymbol{O}\left({ }^{1} \mathrm{~S}\right)}}$ \\
\hline 2015 & 5.91 & 0.26 & 4.91 & 0.13 \\
2016 & 5.48 & 0.29 & 4.98 & 0.07 \\
2017 & 5.03 & 0.44 & 4.76 & 0.24 \\
\hline
\end{tabular}

Note that the asymptotic value of the modeled CF is just the value of CF for very large $\lambda_{z}$ in Figure 3. At this large scale range, CF tends to a stationary, unchanged value of 3.5 (5.1) for the $\mathrm{OH}\left(\mathrm{O}\left({ }^{1} \mathrm{~S}\right)\right)$ emission. Thus, a measure of the discrepancy between modeled and observed CF takes into account the asymptotic value of the modeled CF of both layers. The calculated discrepancy is a simple way to provide an empirical correction to the modeled CF for the emissions, although further investigation into the CF model assumptions and parameters must be carried out. The corrected CF model curves are shown in Figure 5.
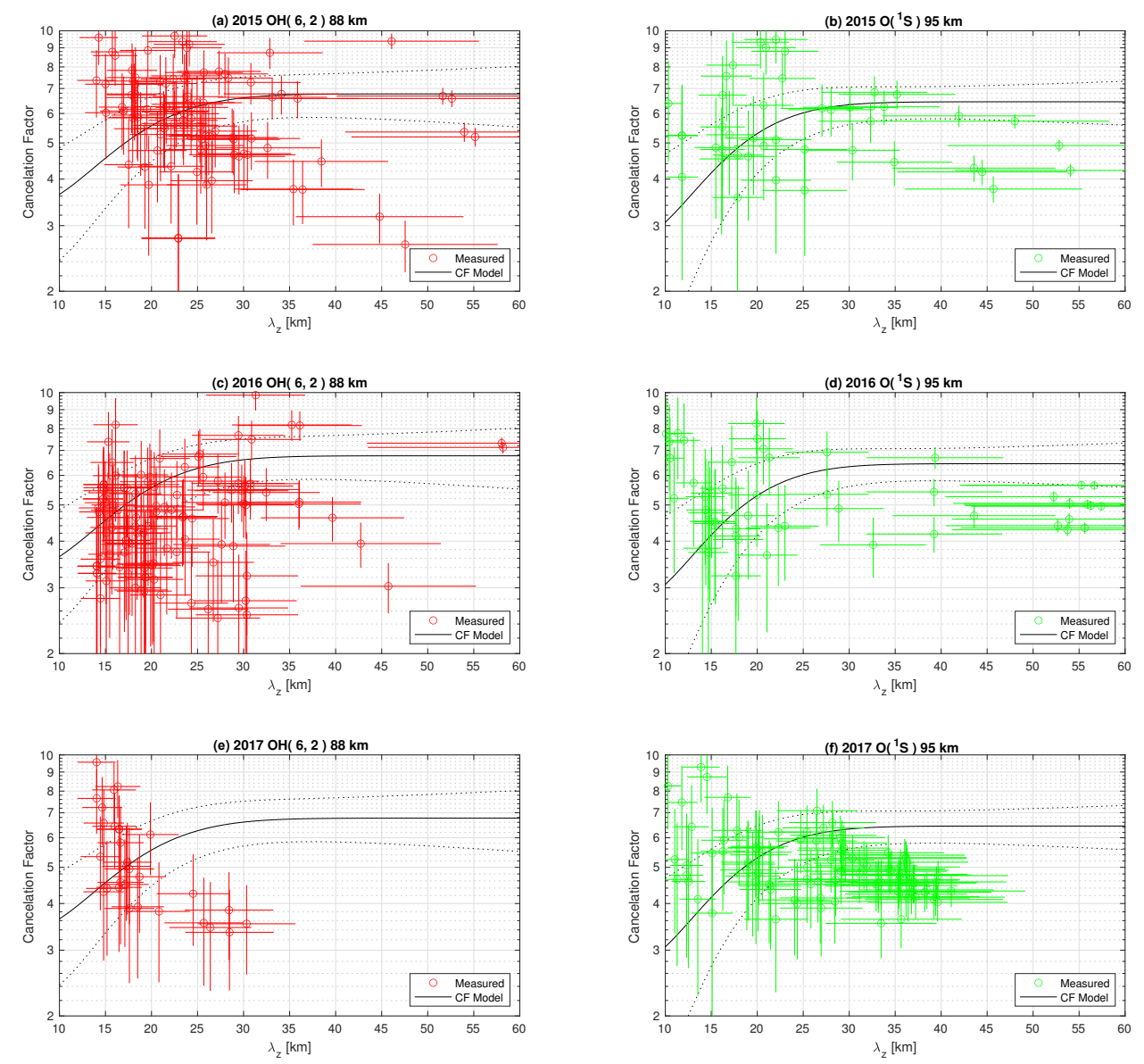

Figure 5. Observed cancellation factor corrected for both $O H$ (red open circles) during (a) 2015, (c) 2016, and (e) 2017, and for $O\left({ }^{1} S\right)$ (green open circles) during (b) 2015, (d) 2016, and (f) 2017 along with their errors $\sigma_{\lambda_{z}}$ and $\sigma_{C F}$. The dotted thin lines denote the $95 \%$ confidence bounds around the analytic curves shown as continuous black lines. 
The CF weighted mean and weighted errors computed for the $O\left({ }^{1} S\right)$ emission lines in 2015, 2016, and 2017 are in good agreement with the modeled asymptotic value, $\mathrm{CF}_{O\left({ }^{1} S\right)} \sim 4.5$ for large values of $\lambda_{z}$. However, we did not find a good result for the $\mathrm{CF}_{O H} \sim=3.5$ asymptotic value, as the estimated weighted mean was much larger than in the model in the high wavelength range.

\section{Discussion}

We tested the modeled CF presented in [21] for the Meinel $O H$ band emission and $O\left({ }^{1} S\right)$ emission line using observed data obtained from ALO. We reported perturbations in the nightglow intensity in response to the AGWs under cancellation effects modeled with an empirical method that considers a windless and isothermal atmosphere with upward propagating and saturated waves (the wave amplitude does not change with altitude). Figure 3 shows the cancellation factors in both layers as functions of $\lambda_{z}$. From the definition, we see that smaller CF corresponds to a stronger cancellation effect. Conversely, $\mathrm{CF}$ increases with increasing $\lambda_{z}$ up to an asymptotic value above the unit, showing that the wave amplitude is amplified by the layer response to the wave perturbation.

The intensity perturbations with small vertical scale $\left(\lambda_{z}<10 \mathrm{~km}\right)$ have strong cancellation in the layer because of the finite thickness of the nightglow layers, which implies that these short $\lambda_{z}$ waves do not show significant amplitudes from ground observations ([20]). Thus, the nightglow is not sensitive to these waves. Equation (11) presented in [26] shows that the analytical function describing $\mathrm{CF}$ increases monotonically with $\lambda_{z}>13 \mathrm{~km}$ for $\mathrm{OH}$ band emission and $\lambda_{z}>10 \mathrm{~km}$ for the $O\left({ }^{1} \mathrm{~S}\right)$ emission line; therefore, for $\lambda_{z}$ lower than these limits the cancellation effect gets stronger.

The centroid height and thickness (FWHM) of the unperturbed and standard deviations of the VER profiles derived for the $O H$ layer are larger than that the $O\left({ }^{1} S\right)$ layer (see Table 1 in [21]), which results in a stronger cancellation effect in the $\mathrm{OH}$ layer and therefore the $\mathrm{CF}$ for $\mathrm{O}\left({ }^{1} \mathrm{~S}\right)$ emission is larger than that for $\mathrm{OH}$, indicating that the greenline nightglow is more sensitive to AGWs. For $\lambda_{z}$ larger than $\sim 20 \mathrm{~km}$, the layer thickness becomes irrelevant because the layer thickness is a fraction of the vertical wavelength; the layer response is stronger and virtually the same for longer vertical wavelength waves.

The work of [26] has presented a comprehensive discussion about the magnitude of the uncertainties in gravity wave parameters estimated from nightglow measurements, and how these uncertainties affect the estimation of key dynamic quantities in the mesosphere and lower thermosphere region. In this study, we derived the uncertainties in CFs and vertical wavelengths, which are subject to large uncertainties. However, these magnitudes are in agreement with conclusions reported in [26].

A source of discrepancy between the modeled and observed CF values found in this study is that the CF model considers saturated waves only. In a real atmosphere, saturated waves co-exist with dissipative and freely propagating waves. That fact likely accounts for the majority of the discrepancy in our results because we have not separated waves by their kind in this study. As all observed wave cases go into our analysis for comparisons with the CF model, we cannot guarantee that the observed waves are saturated waves as in the CF model.

Another source of discrepancy in our results with the modeled $\mathrm{CF}$ for the $\mathrm{OH}$ and $O\left({ }^{1} S\right)$ layers is relative to the distribution of atomic oxygen with height in the presence of vertically propagating waves, which could also influence the results here. The waves influence the temperature gradient that affects the rate of chemical reactions in the nightglow emissions ([18]). The distribution of species involved in nightglow emissions varies considerably with latitude and time, constituting another source of discrepancy between model and measurements ([32]) once the model considers only calm, low solar cycle atmospheric conditions.

In addition, based on a full-wave model with the relevant chemistry for the nightglow emissions that considers more physical processes such as propagating gravity waves in a non-isothermal mean state, and windy (background winds $\neq 0$ as a function of height) and viscous atmospheres, the cancellation factors can vary considerably by a factor of two greater than isothermal and windless 
values for gravity waves of short horizontal wavelengths with phase velocities less than $100 \mathrm{~m} / \mathrm{s}$, and by a factor of one hundred for phase speeds less than $40 \mathrm{~m} / \mathrm{s}$ as reported in [32].

All in all, having tested the modeled CF relationship against observed data for two nightglow layers, we have found that the modeled CF underestimates the observations for both emissions. The cancellation effect is found to be larger in magnitude for $\mathrm{OH}$-band emission than for the $\mathrm{O}\left({ }^{1} \mathrm{~S}\right)$ emission line. However, $\mathrm{CF}$ is still a valuable parameter for retrieving the magnitude of the relative temperature fluctuation from the nightglow, which is used to estimate the momentum flux magnitude transported by the waves ([26]).

The impact of the empirical correction is most significant on momentum flux estimations for the $\mathrm{OH}$ emission. For instance, [33] estimated the vertical flux of horizontal momentum associated with an extensive and bright mesospheric gravity wave event that occurred over the El Leoncito Observatory, Argentina ( $\left.31.8^{\circ} \mathrm{S}, 69.3^{\circ} \mathrm{W}\right)$, during the nights of 17 and 18 March 2016. The estimated average momentum flux of this spectacular wave was $\sim 232 \mathrm{~m}^{2} / \mathrm{s}^{2}$ using the modeled CF of [21], but according to our correction, this estimated value would be reduced by a factor of 3.4 as the momentum flux depends on $\mathrm{CF}^{-2}$.

\section{Conclusions}

We used observed data from nightglow images and lidar temperature and winds to derive the observed cancellation factor for comparison with the modeled CF. We quantified the perturbations generated by gravity waves detected in the $\mathrm{OH}$ and $O\left({ }^{1} S\right)$ nightglow imagery data taken at ALO from 2015 to 2017. We have provided a long-term study in calculating the magnitude of the cancellation factor, fundamental intrinsic wave parameters, and their uncertainties estimated for different seasonal environment scenarios, as well as different background conditions provided by the upper atmosphere climatological models (NRLMSISE-00 model) for $\mathrm{OH}$ and $\mathrm{O}\left({ }^{1} S\right)$ emission. The main results of this work are as follows.

1. We found that the modeled CF relationship underestimates the observations as shown in Figure 3. The discrepancies might have come from that dissipative and freely propagating waves co-exist with saturated waves (as hypothesised in the CF model). However, we have not separated waves by their kind in this study. That is due to that we did not measure waves simultaneously in different layers, which would be the only way to determine how the wave amplitude changers as it moves upwards. Another possible source of discrepancies could be introduced by the photochemical scheme used to model the cancellation factor. The model does not use realistic atomic oxygen data (see [21]) to obtain the CF magnitude. As the atomic oxygen density is affected by the season and the solar cycle activity, one way to improve the model and its agreement with the observations is to have the $O$ density determined individually for each observation night to take into account the season and solar cycle conditions. Beyond that, we believe that the distribution of atomic oxygen with height in the presence of vertically propagating waves is influenced by a temperature gradient that affects the rate of chemical reactions of the nightglow emissions ([18]), which would also contribute to the discrepancies. By accounting for these discrepancy sources, it will be possible to improve the CF model for both studied nightglow layers.

2. Because the modeled CF underestimates the observed $\mathrm{CF}$, we have performed a correction in the modeled $\mathrm{CF}$ curve by estimating the discrepancies from the observed wave data obtained from both $O H$ and $O\left({ }^{1} S\right)$ emissions. We used the weighted mean and weighted standard deviation to provide a measure of the discrepancy between modeled and observational CFs in Table 6.

3. We have adjusted the modeled CF by the observed CF weighted mean to obtain an empirical correction for the modeled $\mathrm{CF}$ for both $\mathrm{OH}$ and $\mathrm{O}\left({ }^{1} S\right)$ emissions. However, the observations still deviate by a factor of $\sim 2$ for the modeled $\mathrm{CF}_{\mathrm{OH}}$, showing that this simpler correction does not work for the hydroxyl emission. Therefore, another strategy must be devised to improve the model and the agreement between the model and the observations. 
4. Despite these discrepancies, there are consistencies in the range $20<\lambda_{z}<60 \mathrm{~km}$ between the modeled and observed CF relationships based on the observed CF error bars that fall into the $95 \%$ confidence levels of the modeled $\mathrm{CF}$, mainly for the $\mathrm{O}\left({ }^{1} S\right)$ layer.

Author Contributions: Methodology, F.V., P.V.; software, L.N., J.F, and F.V.; formal analysis, F.V., J.F.; investigation, J.F.; resources, F.V., G.S.; writing — original draft preparation, J.F.; writing—review and editing, J.F., F.V. All authors have read and agreed to the published version of the manuscript.

Funding: This research has been supported by the National Science Foundation under 1-NSF AGS Grant \#17-59573 and 2-NSF AGS Grant \#19-03336.

Acknowledgments: We are grateful to Luis Navarro who provided us with the source code for nightglow image processing. We also thank two anonymous referees for their pertinent comments and suggestions to improve this paper.

Conflicts of Interest: The authors of this article declare no conflicts of interest.

\section{References}

1. Walterscheid, R.L.; Schubert, G. Gravity wave fluxes of $\mathrm{O}_{3}$ and $\mathrm{OH}$ at the nightside mesopause. Geophys. Res. Lett. 1989, 16, 719-722. [CrossRef]

2. Hickey, M.P.; Walterscheid, R.L.; Richards, P.G. Secular variations of atomic oxygen in the mesopause region induced by transient gravity wave packets. Geophys. Res. Lett. 2000, 27, 3599-3602. [CrossRef]

3. MacDougall, J.; Plane, J.; Jayachandran, P. Polar cap Sporadic-E: Part 2, modeling. J. Atmos. Sol. Terr. Phys. 2000, 62, 1169- 1176. [CrossRef]

4. Cai, X.; Yuan, T.; Eccles, J.V. A Numerical Investigation on Tidal and Gravity Wave Contributions to the Summer Time Na Variations in the Midlatitude E Region. J. Geophys. Res. Space Phys. 2017, 122, 10,577-10,595. [CrossRef]

5. Cai, X.; Yuan, T.; Eccles, J.V.; Raizada, S. Investigation on the Distinct Nocturnal Secondary Sodium Layer Behavior Above $95 \mathrm{~km}$ in Winter and Summer Over Logan, UT $\left(41.7^{\circ} \mathrm{N}, 112^{\circ} \mathrm{W}\right)$ and Arecibo Observatory, PR $\left(18.3^{\circ} \mathrm{N}, 67^{\circ}\right.$ W). J. Geophys. Res. Space Phys. 2019, 124, 9610-9625. [CrossRef]

6. Hecht, J.H.; Walterscheid, R.L.; Kane, T.J.; Gardner, C.S.; Tepley, C.A. Simultaneous nightglow and Na lidar observations at Arecibo during the AIDA-89 campaign. J. Atmos. Terr. Phys. 1993, 55, 409-423. [CrossRef]

7. Hecht, J.H.; Walterscheid, R.L.; Ross, M.N. First measurements of the two-dimensional horizontal wave number spectrum from CCD images of the nightglow. J. Geophys. Res. 1994, 99, 11449-11460. [CrossRef]

8. Swenson, G.R.; Taylor, M.J.; Espy, P.J.; Gardner, C.; Tac, X. ALOHA-93 measurements of intrinsic AGW characteristics using airborne airglow imager and groundbased Na wind/temperature lidar. Geophys. Res. Lett. 1995, 22, 2841-2844. [CrossRef]

9. Smith, S.M.; Mendillo, M.; Baumgardner, J.; Clark, R.R. Mesospheric gravity wave imaging at a subauroral site: First results from Millstone Hill. J. Geophys. Res. 2000, 105, 27119-27130. [CrossRef]

10. Yu, J.R.; Latifi, H.; She, C.Y.; Yee, J.H.; Niciejewski, R.J. Simultaneous lidar and airglow temperature measurements in the mesopause region. Geophys. Res. Lett. 1991, 18, 1361-1363. [CrossRef]

11. Hecht, J.H.; Walterscheid, R.L. Observations of the $\mathrm{OH}$ Meinel $(6,2)$ and $\mathrm{O}_{2}$ atmospheric $(0,1)$ Nightglow emissions from Maui during the ALOHA-90 Campaign. Geophys. Res. Lett. 1991, 18, 1341-1344. [CrossRef]

12. Krassovsky, V.I. Infrasonic variations of OH-emission in upper-atmosphere. Ann. Geophys. 1972, 28, 739-746.

13. Walterscheid, R.L.; Schubert, G.; Straus, J.M. A dynamical-chemical model of wave-driven fluctuations in the OH nightglow. J. Geophys. Res. Space Phys. 1987, 92, 1241-1254. [CrossRef]

14. Hickey, M.P. Effects of eddy viscosity and thermal conduction and Coriolis force in the dynamics of gravity wave driven fluctuations in the OH nightglow. J. Geophys. Res. Space Phys. 1988, 93, 4077-4088. [CrossRef]

15. Tarasick, D.W.; Shepherd, G.G. Effects of gravity waves on complex airglow chemistries: 2. OH emission. J. Geophys. Res. Space Phys. 1992, 97, 3195-3208. [CrossRef]

16. Makhlouf, U.B.; Picard, R.H.; Winick, J.R. Photochemical-dynamical modeling of the measured response of airglow to gravity waves: 1. Basic model for OH airglow. J. Geophys. Res. Atmos. 1995, 100, 11289-11311. [CrossRef]

17. Zhang, S.; Wiens, R.; Shepherd, G. Gravity waves from $\mathrm{O}_{2}$ nightglow during the AIDA ' 89 campaign II: numerical modeling of the emission rate/temperature ratio, $\eta$. J. Atmos. Terr. Phys. 1993, 55, 377- 395. [CrossRef] 
18. Swenson, G.R.; Gardner, C.S. Analytical models for the responses of the mesospheric $\mathrm{OH}^{*}$ and Na layers to atmospheric gravity waves. J. Geophys. Res. 1998, 103, 6271-6294. [CrossRef]

19. Swenson, G.R.; Liu, A.Z. A model for calculating acoustic gravity wave energy and momentum flux in the mesosphere from OH airglow. Geophys. Res. Lett. 1998, 25, 477-480. [CrossRef]

20. Liu, A.Z.; Swenson, G.R. A modeling study of $\mathrm{O}_{2}$ and $\mathrm{OH}$ airglow perturbations induced by atmospheric gravity waves. J. Geophys. Res. (Atmos.) 2003, 108, 4151. [CrossRef]

21. Vargas, F.; Swenson, G.; Liu, A.; Gobbi, D. $\mathrm{O}\left({ }^{1} S\right), \mathrm{OH}$, and $\mathrm{O}_{2}(\mathrm{~b})$ airglow layer perturbations due to AGWs and their implied effects on the atmosphere. J. Geophys. Res. 2007, 112, [CrossRef]

22. Taylor, M.; Hapgood, M.; Rothwell, P. Observations of gravity wave propagation in the OI (557.7 nm), Na $(589.2 \mathrm{~nm})$ and the near infrared $\mathrm{OH}$ nightglow emissions. Planet. Space Sci. 1987, 35, 413- 427. [CrossRef]

23. Swenson, G.R.; Mende, S.B. OH emission and gravity waves (including a breaking wave) in all-sky imagery from Bear Lake, UT. Geophys. Res. Lett. 1994, 21, 2239-2242. [CrossRef]

24. Vincent, R. Gravity-wave motions in the mesosphere. J. Atmos. Terr. Phys. 1984, 46, 119- 128. [CrossRef]

25. Fritts, D.C.; Lu, W. Spectral Estimates of Gravity Wave Energy and Momentum Fluxes. Part II: Parameterization of Wave Forcing and Variability. J. Atmos. Sci. 1993, 50, 3695-3713. [CrossRef]

26. Vargas, F. Uncertainties in gravity wave parameters, momentum fluxes, and flux divergences estimated from multi-layer measurements of mesospheric nightglow layers. Adv. Space Res. 2019, 63, 967-985. [CrossRef]

27. Krueger, D.A.; She, C.Y.; Yuan, T. Retrieving mesopause temperature and line-of-sight wind from full-diurnal-cycle Na lidar observations. Appl. Opt. 2015, 54, 9469-9489. [CrossRef]

28. She, C.Y.; Yu, J.R. Simultaneous three-frequency Na lidar measurements of radial wind and temperature in the mesopause region. Geophys. Res. Lett. 1994, 21, 1771-1774. [CrossRef]

29. Picone, J.M.; Hedin, A.E.; Drob, D.P.; Aikin, A.C. NRLMSISE-00 empirical model of the atmosphere: Statistical comparisons and scientific issues. J. Geophys. Res. (Space Phys.) 2002, 107, 1468. [CrossRef]

30. Garcia, F.J.; Taylor, M.J.; Kelley, M.C. Two-dimensional spectral analysis of mesospheric airglow image data. Appl. Opt. 1997, 36, 7374-7385. [CrossRef]

31. Freedman, D.; Diaconis, P. On the histogram as a density estimator: $\mathrm{L}_{2}$ theory. Z. Wahrscheinlichkeitstheorie Verw Gebiete 1981, 57, 453-476. [CrossRef]

32. Hickey, M.P.; Yu, Y. A full-wave investigation of the use of a "cancellation factor" in gravity wave-OH airglow interaction studies. J. Geophys. Res. (Space Phys.) 2005, 110, A01301. [CrossRef]

33. Smith, S.M.; Setvák, M.; Beletsky, Y.; Baumgardner, J.; Mendillo, M. Mesospheric Gravity Wave Momentum Flux Associated With a Large Thunderstorm Complex. J. Geophys. Res. Atmos. 2020, 125, e2020JD033381. [CrossRef]

Publisher's Note: MDPI stays neutral with regard to jurisdictional claims in published maps and institutional affiliations.

(C) 2020 by the authors. Licensee MDPI, Basel, Switzerland. This article is an open access article distributed under the terms and conditions of the Creative Commons Attribution (CC BY) license (http:/ / creativecommons.org/licenses/by/4.0/). 